\title{
Timing of Liver Surgery for Colorectal Liver Metastases will be Influenced by Tumor Behavior
}

\author{
François Cauchy, MD and Jacques Belghiti, MD \\ HPB Surgery \& Liver Transplantation, Beaujon Hospital [Assistance Publique-Hôpitaux de Paris], University Paris, Paris, \\ France
}

We all know that surgery remains the best chance of cure for patients with colorectal liver metastases (CLM). Within 2 years following resection, half of the patients will experience recurrence, but it is commonly accepted that repeat resection, when technically feasible, provides excellent long-term outcomes, joining that of patients without recurrence. ${ }^{1}$ One should nevertheless bear in mind that these prolonged survivals are often the result of the multimodal management of the patients, which combines sophisticated morphological follow-up, percutaneous ablative therapies and, above all, systemic chemotherapy.

In the current study, Vigano et $\mathrm{al}^{2}{ }^{2}$ confirm these previous findings but also provide new insights regarding the pattern of recurrences of patients operated for CLM. Using rigorous data arising from the well-known LiverMetSurvey registry, they have specifically focused on the $10 \%$ of patients experiencing early ( $<6$ months) recurrence following resection of CLM. Not surprisingly, the authors observed that such recurrences were more likely to occur in patients with pejorative factors, including advanced primary disease, synchronous metastatic spread, high number of lesions, surgical margin invasion, and absence of adjuvant chemotherapy. Also, they established that early recurrences were associated with impaired long-term prognosis, especially when not amenable to repeated resection. While these valuable findings favor an all oncosurgical approach and are in line with what might have been expected, several important key aspects still remain to be discussed.

\footnotetext{
(C) Society of Surgical Oncology 2013
}

First Received: 18 November 2013;

Published Online: 6 December 2013

J. Belghiti, MD

e-mail: Jacques.belghiti@bjn.aphp.fr

\section{WHICH SURGICAL MARGIN?}

Current accurate pathological analysis of the resected specimen mainly focuses on both tumor grading and quantification of necrotic or fibrotic changes within the lesions. Nevertheless, several important histological factors at the tumor-normal liver interface, such as tumor thickness, existence of a peripheral halo composed of viable cells, satellite nodules, as well as lymphatic or microscopic biliary invasion, have recently shown to strongly correlate with recurrence. ${ }^{3}$ These observations support the fact that aiming at only attaining $\mathrm{R} 0$ resection is now irrelevant and should lead us to reconsider classical technical rules in order to obtain wider margins. On one hand, results of the present study confirm that the use of intraoperative ultrasound is of priceless assistance in order to stay at a respectful distance while performing deep resections, ${ }^{4}$ but also justify our policy of intention-to-treat margins of at least $1 \mathrm{~cm}$. On the other hand, the current study only touches the opportunity to revisit the concept of anatomical resection in patients with CLM. Even though this observation revives an old debate, it may appear of particular significance in the specific setting of patients with CLM undergoing preoperative chemotherapy.

\section{WHAT IS THE ROLE OF PREOPERATIVE CHEMOTHERAPY?}

Obviously, an increasing proportion of patients with upfront resectable metastatic disease will be referred while already receiving preoperative chemotherapy. This 'reality check' renders pointless any debate questioning the actual benefits of preoperative chemotherapy. Therefore, surgeons have now to face several other concerns related to its use. First, determining the optimal timing for surgery. Indeed, while planning surgery too early would not allow for 
appropriate control of the metastatic disease, prolonging chemotherapy could lead to metastatic disappearance but also to unnecessary development of chemotherapy-associated liver injury. Thus, development of biological, oncogenetic and morphological relevant markers allowing accurate assessment of tumor response are now required. Second, understanding the dramatic influence of tumor biology. In the current study, response to chemotherapy was a strong prognostic factor for early recurrence. This finding is in line with previous studies emphasizing that time to response to optimal chemotherapy could represent contraindications to major surgery and therefore supports that response to chemotherapy should also be interpreted in light of the natural history of the disease and not only on a purely technical basis. ${ }^{5}$

\section{REFERENCES}

1. Wicherts DA, de Haas RJ, Salloum C, Andreani P, Pascal G, Sotirov D, et al. Repeat hepatectomy for recurrent colorectal metastases. Br J Surg. 2013;100:808-18.
2. Viganò L, Capussotti L, Lapointe R, Barroso E, Hubert C, Giuliante $\mathrm{F}$, et al. Early recurrence after liver resection for colorectal metastases: risk factors, prognosis, and treatment. A LiverMetSurvey-based study of 6025 patients. Ann Surg Oncol. doi:10.1245/s10434-013-3421-8.

3. Mentha G, Terraz S, Morel P, Andres A, Giostra E, Roth A, et al. Dangerous halo after neoadjuvant chemotherapy and two-step hepatectomy for colorectal liver metastases. $\mathrm{Br} J$ Surg. 2009;96:95-103.

4. Torzilli G, Montorsi M, Donadon M, Palmisano A, Del Fabbro D, Gambetti A, et al. "Radical but conservative" is the main goal for ultrasonography-guided liver resection: prospective validation of this approach. J Am Coll Surg. 2005;201:517-28.

5. Cauchy F, Aussilhou B, Dokmak S, Fuks D, Gaujoux S, Farges O, et al. Reappraisal of the risks and benefits of major liver resection in patients with initially unresectable colorectal liver metastases. Ann Surg. 2012;256:746-52. 